\title{
DETERMINING THE REFERENCE EPHEMEROPTERA COMMUNITIES IN THE EASTERN PART OF THE BLACK SEA REGION FOR THE IMPLEMENTATION OF THE WATER FRAMEWORK DIRECTIVE IN TURKEY
}

\author{
Gencer TÜRKMEN * and Nilgün KAZANCI *
}

* Hacettepe University, Department of Biology, Biomonitoring Laboratory, Hydrobiology Section, Beytepe Campus, Ankara, Turkey, TR-06800, gencerturkmen@gmail.com, nilgunkazanci@gmail.com

DOI: 10.1515/trser-2015-0058

KEYWORDS: Aquatic insects, benthic macroinvertebrate, biodiversity, Black Sea, Ephemeroptera, references site, reference community, Turkey, water framework directive.

\section{ABSTRACT}

In this study, we aimed to determine the reference sites and their reference Ephemeroptera communities according to the Water Framework Directive methods in the Eastern Part of the Black Sea Region of Turkey between 2008-2011. From the area, twentyfour collecting sites were chosen. There were thirty Ephemeroptera species identified, belonging to seven families and eleven genres. Cluster analysis based on Bray-Curtis similarities was applied. Reference habitat conditions of the studied sites and their reference Ephemeroptera communities were determined by combining both ecological and statistical results. As a result, sixteen sites had reference habitat conditions and their reference Ephemeroptera species were identified.

RÉSUMÉ: Déterminer les communautés de référence des Ephéméroptères dans la partie est de la région de la Mer Noire pour implémenter la Directive Cadre de l'Eau en Turquie.

Dans cette étude, nous avons cherché à déterminer les sites de référence et les communautés de référence d'Ephemeroptera selon les méthodes préfigurées par la Directive Cadre de l'Eau dans la partie orientale de la région de la Mer Noire de la Turquie entre 20082011. Vingt-quatre sites d'échantillonnage ont été choisis dans la région. Une trentaine d'espèces d'Ephemeroptera ont été identifiées, appartenant à sept familles et onze genres. L'analyse de cluster basée sur des similitudes de Bray-Curtis a été appliquée. Les conditions d'habitat de référence des sites ont été étudiées et leurs communautés de référence des Ephemeroptera ont été déterminées en combinant à la fois les résultats écologiques et statistiques. En conséquence, seize sites présentaient des conditions d’habitat de référence et leurs espèces Ephemeroptera de référence ont été identifiées.

REZUMAT: Determinarea comunităţilor de efemeroptere de referinţă, în partea estică a regiunii Mării Negre pentru implementarea Directivei Cadru Apă în Turcia.

Acest studiu are ca scop identificarea siturilor de referinţă conform Directivei Cadru Apa, din perspectiva structurii comunităţilor de efemeroptere şi a habitatelor carecteristice pentru acestea, din partea de Est a regiunii Mării Negre a Turciei. Studiul s-a derulat în perioada 2008-2011. Au fost alese 24 de staţii de prelevare a probelor din aria de referință. S-au identificat 30 de specii de efemeroptere, aparținând la şapte familii şi unsprezece genuri și s-a aplicat analiza Cluster bazată pe similaritățile Bray-Curtis. Datele ecologice și statistice au fost utilizate pentru a determina condițiile de habitat și structura comunităţilor de referinţă. Rezultatul arată că şaisprezece dintre situri au respectat condițiile de habitat pentru speciile de efemeroptere, în aceste situri au fost identificate speciile indicatoare de stare favorabilă a sistemului acvatic, cu succes. 


\section{INTRODUCTION}

The European Union Water Framework Directive (WFD) is a legislation which aims to achieve at least a good ecological quality of all surface and groundwater ecosystems for all member states of the union by 2015 (Council of European Communities, 2000). According to this directive, all member states have to change their water management plans, especially on river basins. Aquatic ecology and the reference site concept are on the basis of these management plans in WFD (Council of European Communities, 2000). It is emphasized that water is not a commercial product, it is a necessary source that should be cared for and protected. The aims of the WFD could be summarized as: prevention of devastation on aquatic ecosystems, improvement of aquatic ecosystems' health, and long-term protection of the present water resources (Van Wijk et al., 2003).

The concept of "River Basin Management" is a new and very important approach for the member states of the EU because water bodies are not evaluated according to the governmental and political borders, but rather divided into river basins according to natural, geographical and hydrological basis (Çoşkun, 2010).

Turkey is conducting accession negotiations with the EU as the environment constitutes one of the significant areas in aquis communautaire. Turkey, as a candidate country, is obliged to get in line with the WFD in time for membership. In this concept, there have been many studies carried out by the Ministry of Forestry and Water Affairs of Turkey. Twenty-five river basins were specified in Turkey, and the Eastern Part of the Black Sea Region is one of them.

Benthic macroinvertebrates, especially aquatic insects, have great importance in aquatic ecology (Rosenberg and Resh, 1993; Clarke et al., 2013). Aquatic insects can be found in almost all kinds of freshwater ecosystems. Many orders are sensitive to water quality such as Ephemeroptera, Plecoptera, Odonata, Trichoptera, Coleoptera, etc. Ephemeroptera is the most important insect order in running water ecosystems (Bănăduc and Olosutean, 2013; Buffagni et al., 2001; Moog et al., 1997). There are many indicator species of clean and undegraded freshwater habitats in this order (Metcalfe, 1989; Hubbard and Peters, 1978). Many of them are very sensitive to organic pollution, physical degradation and habitat loss.

In this study, the Eastern Part of the Black Sea Region of Turkey was chosen; the reason being that there are several mountain running waters in this region and Ephemeroptera fauna of this area is very rich compared to the other parts of Turkey. This region is also important because of the Caucasian Biodiversity Hotspot (Kazanc1, 2013; Türkmen and Kazanc1, 2015; Kazanc1 et al., 2011, 2013). The running waters in this region are generally unpolluted and many of them can be accepted as reference sites. However, investments and activities in tourism have recently been increasing in this region. Increasing tourism activities are starting to bring urbanization as well. Furthermore, and possibly the most devastating, there are many hydroelectric power plants already constructed on some running waters and many of them are planned for construction.

All of these factors are affecting the area in terms of the loss of aquatic habitats, reference sites and aquatic communities, (especially the Ephemeroptera species). Many Ephemeroptera species may face the danger of extinction. For this reason, determining the reference Ephemeroptera communities in this region has a great importance for the future of aquatic ecosystem studies. The knowledge about these communities gives us an opportunity for the comparative studies and the preparation of biotic indices for the region. 


\section{MATERIAL AND METHODS}

In this research, 24 sites were examined in some streams in the Eastern Part of the Black Sea Region of Turkey between 2008 and 2011. Benthic macroinvertebrate samples were collected from these sites by standard kick-net and kept in $4 \%$ formaldehyde solution. After the samples were brought to the laboratory, they were washed and the Ephemeroptera individuals were separated and kept in an 80\% ethyl alcohol solution. All Ephemeroptera individuals were identified to species level.

Habitat characteristics and stream zonation of the sites were given. Cluster analysis (Bray-Curtis Similarity) was applied to compare the sites in terms of their Ephemeroptera species composition and habitat type. Reference habitat conditions of the studied sites and their reference Ephemeroptera communities were determined by combining both ecological and statistical results.

This study is part of the $\mathrm{PhD}$ Thesis, "Systematic and Ecologic Research on the Ephemeroptera Fauna of the Eastern Black Sea Region to Take Part in the Implementation of Water Framework Directive (WFD)” (Türkmen, 2013).

\section{Description of the study area}

In this study, the Eastern part of the Black Sea Region of Turkey was chosen as the study area (Fig. 1), because, there are many mountain running waters in this region and Ephemeroptera fauna of this area is very rich compared to the other parts of Turkey. This region is also important since it is part of the Caucasian Biodiversity Hotspot. The running waters in this region are generally unpolluted and many of them can be accepted as reference sites.

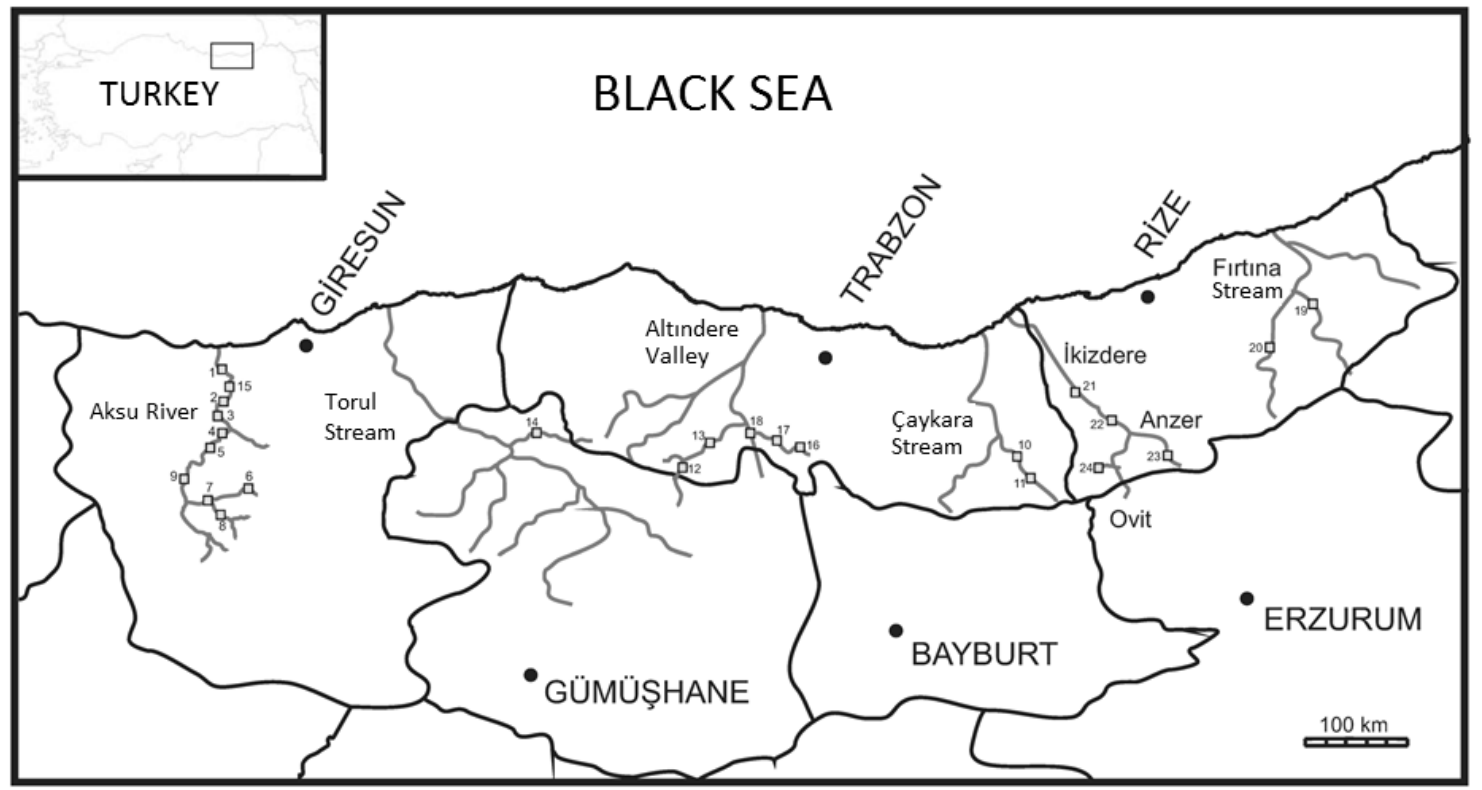

Figure 1: Study area, streams and the location of the sites. 


\section{RESULTS AND DISCUSSION}

In this study, a total of 12,252 Ephemeroptera individuals belonging to seven families, 11 genere and 30 species were identified (Tab. 1). According to the results, the highest genere and species numbers belonged to Heptageniidae with four genere and 17 species, while the highest individual numbers belonged to Baetidae with 7,586 individuals. Percentage distributions of species, genere and individual numbers were shown in table 2. All sites were classified according to System A and System B classification in WFD methods (Tab. 3). In addition to these classification parameters, we added the stream zonation. In table 3 , physical degradation effects and reference habitat conditions were also given.

Table 1: List of the Ephemeroptera families, genera and species.

\begin{tabular}{|c|c|c|}
\hline Family & Genus & Species \\
\hline \multirow[t]{6}{*}{ Baetidae } & Baetis & B. rhodani \\
\hline & & B. gemellus \\
\hline & & B. lutheri \\
\hline & & B. fusca \\
\hline & & B. milani \\
\hline & Alainites & A. muticus \\
\hline \multirow[t]{3}{*}{ Caenidae } & Caenis & C. luctuosa \\
\hline & & C. macrura \\
\hline & & C. martae \\
\hline Leptophlebiidae & Habroleptoides & H. modesta \\
\hline Ephemerellidae & Serratella & S. ignita \\
\hline Ephemeridae & Ephemera & E. danica \\
\hline Potamanthidae & Potamanthus & P. luteus \\
\hline \multirow{17}{*}{ Heptageniidae } & Rhithrogena & $R$ zelinkai \\
\hline & & R. beskidensis \\
\hline & & R. semicolorata \\
\hline & & R. germanica \\
\hline & & R. puytoraci \\
\hline & & R. iridina \\
\hline & Epeorus & E. zaitzevi \\
\hline & & E. alpicola \\
\hline & & E. sylvicola \\
\hline & & E. caucasicus \\
\hline & & E. znojkoi \\
\hline & Ecdyonurus & E. starmachi \\
\hline & & E. macani \\
\hline & & E. helveticus \\
\hline & & E. picteti \\
\hline & Electrogena & E. quadrilineata \\
\hline & & E. affinis \\
\hline
\end{tabular}

Table 2: Percentage distributions of Ephemeroptera genera, species and individuals.

\begin{tabular}{|l|c|c|c|}
\hline 1. Baetidae & 18.2 & 20 & 61.92 \\
\hline 2. Caenidae & 9.1 & 10 & 0.08 \\
\hline 3. Leptophlebiidae & 9.1 & 3.3 & 1.09 \\
\hline 4. Ephemerellidae & 9.1 & 3.3 & 6.20 \\
\hline 5. Ephemeridae & 9.1 & 3.3 & 0.01 \\
\hline 6. Potamanthidae & 9.1 & 3.3 & 0.35 \\
\hline 7. Heptageniidae & 36.4 & 56.7 & 30.35 \\
\hline
\end{tabular}


Table 3: System A and System B classification of sites and their stream zonation, physical degradation effects and reference habitat conditions; Abbreviation: in the headings; S. A., System A; S. B., System B; Alt. Typo., Altitude typology; ER, Ecoregion; Lat., Latitude; Long., Longitude, S. z., Stream zonation; P. d., Physical degradation; RHC, Reference Habitat Condition. In the table; L, Lowland; M - a, Mid - altitude; H, High; M, Medium; Y, Y ecoregion; N, North; E, East; Mp, Metapotamon; Er, Epirhithron; Hc, Hypocrenon; Mr, Metarhithron; Hr, Hyporhithron.

\begin{tabular}{|c|c|c|c|c|c|}
\hline Sites & $\begin{array}{l}\text { Alt. Typo. } \\
\text { (S. A.) }\end{array}$ & $\begin{array}{l}\text { Alt. } \\
\text { (S. B.) }\end{array}$ & $\begin{array}{l}\text { Catchment area } \\
\text { (S. A.) }\end{array}$ & $\begin{array}{c}\text { ER } \\
\text { (S. A.) }\end{array}$ & $\begin{array}{l}\text { Lat. } \\
\text { (S. B.) }\end{array}$ \\
\hline S-1 & $\mathrm{L}(<200 \mathrm{~m})$ & $184 \mathrm{~m}$ & $\mathrm{M}\left(100-1,000 \mathrm{~km}^{2}\right)$ & $\mathrm{Y}$ & $40^{\circ} 46^{\prime} 42.6^{\prime \prime} \mathrm{N}$ \\
\hline $\mathrm{S}-2$ & M-a (200-800 m) & $210 \mathrm{~m}$ & $\mathrm{M}\left(100-1,000 \mathrm{~km}^{2}\right)$ & $\mathrm{Y}$ & $40^{\circ} 46^{\prime} 15.1 ” \mathrm{~N}$ \\
\hline S-3 & $\mathrm{L}(<200 \mathrm{~m})$ & $186 \mathrm{~m}$ & $\mathrm{M}\left(100-1,000 \mathrm{~km}^{2}\right)$ & $\mathrm{Y}$ & $40^{\circ} 46^{\prime} 44.4^{\prime \prime} \mathrm{N}$ \\
\hline S-4 & М-а (200-800 m) & $557 \mathrm{~m}$ & $\mathrm{M}\left(100-1,000 \mathrm{~km}^{2}\right)$ & $\mathrm{Y}$ & $40^{\circ} 41^{\prime} 01.08^{\prime \prime} \mathrm{N}$ \\
\hline S-5 & M-a (200-800 m) & $607 \mathrm{~m}$ & $\mathrm{M}\left(100-1,000 \mathrm{~km}^{2}\right)$ & $\mathrm{Y}$ & $40^{\circ} 40^{\prime} 49.01 ” \mathrm{~N}$ \\
\hline S-6 & $\mathrm{H}(>800 \mathrm{~m})$ & $1,867 \mathrm{~m}$ & $\mathrm{M}\left(100-1,000 \mathrm{~km}^{2}\right)$ & $\mathrm{Y}$ & $40^{\circ} 34^{\prime} 07^{\prime \prime} \mathrm{N}$ \\
\hline S-7 & $\mathrm{H}(>800 \mathrm{~m})$ & $1,581 \mathrm{~m}$ & $\mathrm{M}\left(100-1,000 \mathrm{~km}^{2}\right)$ & $\mathrm{Y}$ & $40^{\circ} 32^{\prime} 31^{\prime \prime} \mathrm{N}$ \\
\hline S-8 & $\mathrm{H}(>800 \mathrm{~m})$ & $1,542 \mathrm{~m}$ & $\mathrm{M}\left(100-1,000 \mathrm{~km}^{2}\right)$ & $\mathrm{Y}$ & $40^{\circ} 32^{\prime} 22^{\prime \prime} \mathrm{N}$ \\
\hline S-9 & $\mathrm{H}(>800 \mathrm{~m})$ & $1,582 \mathrm{~m}$ & $\mathrm{M}\left(100-1,000 \mathrm{~km}^{2}\right)$ & $\mathrm{Y}$ & $40^{\circ} 32^{\prime} 19^{\prime \prime} \mathrm{N}$ \\
\hline S-10 & $\mathrm{H}(>800 \mathrm{~m})$ & $1,256 \mathrm{~m}$ & $\mathrm{M}\left(100-1,000 \mathrm{~km}^{2}\right)$ & $\mathrm{Y}$ & $40^{\circ} 35^{\prime} 48.43^{\prime \prime} \mathrm{N}$ \\
\hline S-11 & $\mathrm{H}(>800 \mathrm{~m})$ & $1,254 \mathrm{~m}$ & $\mathrm{M}\left(100-1,000 \mathrm{~km}^{2}\right)$ & $\mathrm{Y}$ & $40^{\circ} 35^{\prime} 52.39 ” \mathrm{~N}$ \\
\hline S-12 & $\mathrm{H}(>800 \mathrm{~m})$ & $1,944 \mathrm{~m}$ & $\mathrm{M}\left(100-1,000 \mathrm{~km}^{2}\right)$ & $\mathrm{Y}$ & 40³7’21.55” N \\
\hline S-13 & $\mathrm{H}(>800 \mathrm{~m})$ & $1,857 \mathrm{~m}$ & $\mathrm{M}\left(100-1,000 \mathrm{~km}^{2}\right)$ & $\mathrm{Y}$ & $40^{\circ} 39^{\prime} 50.69 ” \mathrm{~N}$ \\
\hline S-14 & $\mathrm{H}(>800 \mathrm{~m})$ & $1,658 \mathrm{~m}$ & $\mathrm{M}\left(100-1,000 \mathrm{~km}^{2}\right)$ & $\mathrm{Y}$ & $40^{\circ} 40^{\prime} 32.34 ” \mathrm{~N}$ \\
\hline S-15 & $\mathrm{L}(<200 \mathrm{~m})$ & $55 \mathrm{~m}$ & $\mathrm{M}\left(100-1,000 \mathrm{~km}^{2}\right)$ & $\mathrm{Y}$ & $40^{\circ} 50^{\prime} 67.5^{\prime \prime} \mathrm{N}$ \\
\hline S-16 & $\mathrm{H}(>800 \mathrm{~m})$ & $2,394 \mathrm{~m}$ & $\mathrm{M}\left(100-1,000 \mathrm{~km}^{2}\right)$ & $\mathrm{Y}$ & 40³6’06.7” N \\
\hline S-17 & $\mathrm{H}(>800 \mathrm{~m})$ & $2,128 \mathrm{~m}$ & $\mathrm{M}\left(100-1,000 \mathrm{~km}^{2}\right)$ & $\mathrm{Y}$ & 40³5’99.7” N \\
\hline S-18 & $\mathrm{H}(>800 \mathrm{~m})$ & $1,919 \mathrm{~m}$ & $\mathrm{M}\left(100-1,000 \mathrm{~km}^{2}\right)$ & $\mathrm{Y}$ & $40^{\circ} 37^{\prime} 37.2^{\prime \prime} \mathrm{N}$ \\
\hline S-19 & M-a (200-800 m) & $568 \mathrm{~m}$ & $\mathrm{M}\left(100-1,000 \mathrm{~km}^{2}\right)$ & $\mathrm{Y}$ & $41^{\circ} 01 ’ 32^{\prime \prime} \mathrm{N}$ \\
\hline S-20 & M-a (200-800 m) & $495 \mathrm{~m}$ & $\mathrm{M}\left(100-1,000 \mathrm{~km}^{2}\right)$ & $\mathrm{Y}$ & 4059’03.6” N \\
\hline S-21 & M-a (200-800 m) & 450 & $\mathrm{M}\left(100-1,000 \mathrm{~km}^{2}\right)$ & $\mathrm{Y}$ & $40^{\circ} 48^{\prime} 59^{\prime \prime} \mathrm{N}$ \\
\hline S-22 & М-а (200-800 m) & $560 \mathrm{~m}$ & $\mathrm{M}\left(100-1,000 \mathrm{~km}^{2}\right)$ & $\mathrm{Y}$ & $40^{\circ} 42^{\prime} 48^{\prime \prime} \mathrm{N}$ \\
\hline S-3 & $\mathrm{H}(>800 \mathrm{~m})$ & $1,970 \mathrm{~m}$ & $\mathrm{M}\left(100-1,000 \mathrm{~km}^{2}\right)$ & $\mathrm{Y}$ & $40^{\circ} 36^{\prime} 07.8 ” \mathrm{~N}$ \\
\hline S-24 & $\mathrm{H}(>800 \mathrm{~m})$ & $2,679 \mathrm{~m}$ & $\mathrm{M}\left(100-1,000 \mathrm{~km}^{2}\right)$ & $\mathrm{Y}$ & $40^{\circ} 37^{\prime} 55.8^{\prime \prime} \mathrm{N}$ \\
\hline
\end{tabular}


Table 3 (continued): System A and System B classification of sites and their stream zonation, physical degradation effects and reference habitat conditions; Abbreviation: In the headings; S. A., System A; S. B., System B; Alt. Typo., Altitude typology; ER, Ecoregion; Lat., Latitude; Long., Longitude, S. z., Stream zonation; P. d., Physical degradation; RHC, Reference Habitat Condition. In the table; L, Lowland; M - a, Mid - altitude; H, High; M, Medium; Y, Y ecoregion; N, North; E, East; Mp, Metapotamon; Er, Epirhithron; Hc, Hypocrenon; Mr, Metarhithron; Hr, Hyporhithron.

\begin{tabular}{|c|c|c|c|c|c|}
\hline Sites & $\begin{array}{l}\text { Long. } \\
\text { (S. B.) }\end{array}$ & $\begin{array}{l}\text { Jeology } \\
\text { (S. A.) }\end{array}$ & S. z. & P. d. & RHC \\
\hline S-1 & $38^{\circ} 26^{\prime} 35.6^{\prime \prime} \mathrm{E}$ & Siliceous & $\mathrm{Mp}$ & + & - \\
\hline S-2 & 38²6’23.7” E & Siliceous & $\mathrm{Mp}$ & + & - \\
\hline S-3 & $38^{\circ} 26^{\prime} 38.8^{\prime \prime} \mathrm{E}$ & Siliceous & $\mathrm{Mp}$ & + & - \\
\hline S-4 & $38^{\circ} 26^{\prime} 29.71^{\prime \prime} \mathrm{E}$ & Siliceous & $\mathrm{Er}$ & - & $\checkmark$ \\
\hline S-5 & $38^{\circ} 26^{\prime} 41^{\prime \prime} \mathrm{E}$ & Siliceous & $\mathrm{Er}$ & - & $\checkmark$ \\
\hline S-6 & $38^{\circ} 28^{\prime} 67^{\prime \prime} \mathrm{E}$ & Siliceous & $\mathrm{Er}$ & - & $\checkmark$ \\
\hline S-7 & $38^{\circ} 23^{\prime} 40^{\prime \prime} \mathrm{E}$ & Siliceous & Er & - & $\checkmark$ \\
\hline S-8 & $38^{\circ} 24^{\prime} 06^{\prime \prime} \mathrm{E}$ & Siliceous & $\mathrm{Er}$ & - & $\checkmark$ \\
\hline S-9 & $38^{\circ} 21^{\prime} 35^{\prime \prime} \mathrm{E}$ & Siliceous & Hc & - & $\checkmark$ \\
\hline S-10 & 40¹9’17.60” E & Siliceous & $\mathrm{Er}$ & - & $\checkmark$ \\
\hline S-11 & $40^{\circ} 19^{\prime} 20.31^{\prime \prime} \mathrm{E}$ & Siliceous & Hc & - & $\checkmark$ \\
\hline S-12 & 39³5’37.67” E & Siliceous & Hc & - & $\checkmark$ \\
\hline S-13 & $39^{\circ} 35^{\prime} 55.05^{\prime \prime} \mathrm{E}$ & Siliceous & $\mathrm{Er}$ & - & $\checkmark$ \\
\hline S-14 & $39^{\circ} 25^{\prime} 04.30^{\prime \prime} \mathrm{E}$ & Siliceous & $\mathrm{Mr}$ & - & $\checkmark$ \\
\hline S-15 & $38^{\circ} 27^{\prime} 62.3^{\prime \prime} \mathrm{E}$ & Siliceous & Mp & + & - \\
\hline S-16 & 39³8’42.8” E & Siliceous & Hc & + & - \\
\hline S-17 & $39^{\circ} 37^{\prime} 00^{\prime \prime} \mathrm{E}$ & Siliceous & $\mathrm{Er}$ & + & - \\
\hline S-18 & 39³5’63.8” E & Siliceous & Er & + & - \\
\hline S-19 & $41^{\circ} 02^{\prime} 48^{\prime \prime} \mathrm{E}$ & Siliceous & $\mathrm{Mr}$ & - & $\checkmark$ \\
\hline S-20 & $40^{\circ} 57^{\prime} 82.2^{\prime \prime} \mathrm{E}$ & Siliceous & $\mathrm{Mr}$ & - & $\checkmark$ \\
\hline $\mathrm{S}-21$ & $40^{\circ} 32^{\prime} 26.21^{\prime \prime} \mathrm{E}$ & Siliceous & $\mathrm{Hr}$ & - & $\checkmark$ \\
\hline $\mathrm{S}-22$ & 40³7’28.42” E & Siliceous & $\mathrm{Hr}$ & - & $\checkmark$ \\
\hline S-23 & 40³1’39.2” E & Siliceous & Hc & - & $\checkmark$ \\
\hline S-24 & $40^{\circ} 47^{\prime} 00^{\prime \prime} \mathrm{E}$ & Siliceous & Er & + & - \\
\hline
\end{tabular}


Similar habitats have similar species composition (Odum and Barrett, 2005). In this respect, similar aquatic habitats have similar Ephemeroptera species compositions. Similarities between collecting sites are determined with the species compositions in these sites. Different species in similar habitats or similar species in different habitats are detected by similarity analysis and these analyses provide important information about distribution of species and their ecological demands (Kocataş, 1992; Ter Braak, 1986).

In this study, Bray-Curtis similarity analysis was applied and a cluster dendogram was generated to demonstrate the proximity relationship between the collecting sites (Fig. 2).

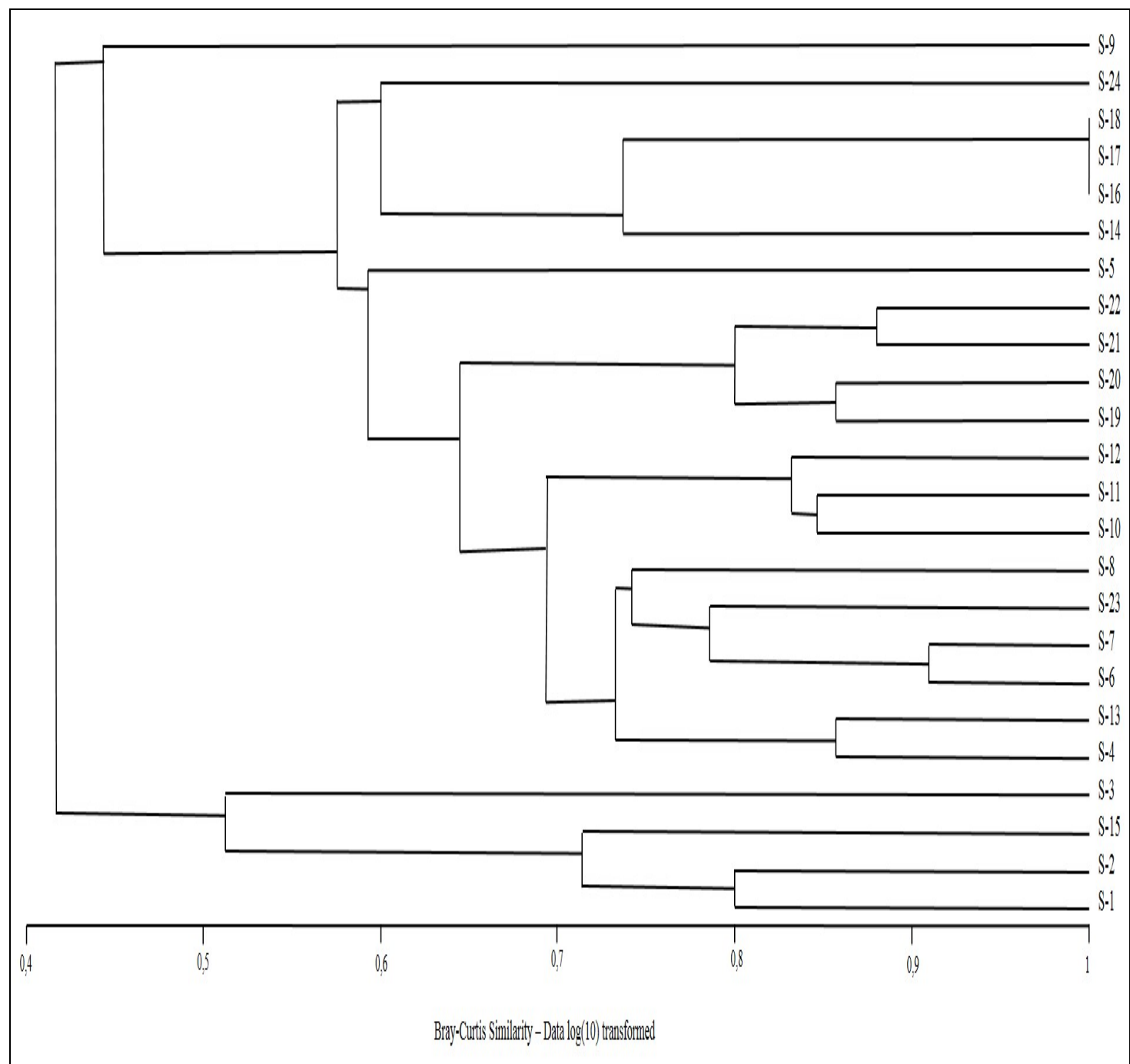

Figure 2: Cluster dendogram of the collecting sites based on Bray-Curtis similarity via Ephemeroptera species composition.

According to the System A and System B classifications, environmental factors and the cluster analysis results, 16 sites have reference habitat conditions while the other eight sites do not due to environmental degradations (Tab. 3). 
S-1, S-2, S-3 and S-15, which did not have reference habitat conditions, differed from the other sites, because of having different typology. As it can be seen in the cluster dendogram, these sites were clustered together. Different typology caused different Ephemeroptera species compositions and this was reflected on the dendogram (Fig. 2).

Different typology conditions are not clearly spotted in System A and System B classification. Thus, stream zonation data of the collecting site(s) were added to help with the decision making. The sites with different typology (S-1, S-2, S-3 and S-15) were situated downstream (metapotamon) (Tab. 3). Environmental characteristics and physico-chemical variables of this zone are different from the upstream zones. These differences lead to different species composition. Caenis martae, C. macrura, C. luctuosa, Ephemera danica, and Potamanthus lutheus were only found in these four sites. Stream zonation preferences of these species are mostly potamon zones (epi-, meta- and hypo-potamon) of running waters (Bauernfeind and Moog, 2002; Buffagni et al., 2009). These species prefer beta-mesosaprobic and alpha-mesosaprobic habitats rather than oligosaprobic and ksenosaprobic habitats (Bauernfeind and Moog, 2002; Buffagni et al., 2009). Saprobic characteristics of the sites where these species were found were beta- and alpha-mesosaprobic (Tab. 4). These species were compatible with not only the stream zonation preferences, but also with the habitat preferences of the sites where they were found in this study. Sites S-1, S-2, S-3 and S-15 were clustered together because they have different typology. In this concept, it would be a mistake to compare these sites to other sites. Therefore, these sites should be considered separately. The reason why these sites did not have reference condition was physical degradations around the sites.

Sites S-16, S-17 and S-18 had similar typology with the other sites, unlike S-1, S-2, S-3 and S-15. Thus, they can be compared to the others. These sites did not have reference habitat conditions (Tab. 3). Also these sites were clustered together and they had the highest similarity ratio according to the Bray-Curtis similarity analysis (Fig. 2). Physical degradations and animal husbandry around the sites caused them to lose reference habitat characteristics. In these sites, Baetidae species (especially B. rhodani) had become quite dominant. In site S-16, dominancy of $B$. rhodani was $45 \%$, entire dominancy of Baetidae species was $97 \%$; in site S-17, dominancy of $B$. rhodani was $46 \%$, entire dominancy of Baetidae species was $94 \%$; in site S-18, dominancy of $B$. rhodani was $46 \%$, entire dominancy of Baetidae species was $89 \%$ (Tab. 5). Moreover, dominancies of the species of Heptageniidae family, which have several clean and undegraded habitat indicator species, were very low in these sites (Tab. 5).

In this study, we also found that the dominancies of Baetidae species were high in non-reference sites, while the dominancies of Heptageniidae species were low. Reversely, the dominancies of Heptageniidae species were high in reference sites, while the dominancies of Baetidae species were low. Only five sites (S-5, S-6, S-9, S11 and S-14) out of twenty-four sites did not fit that result. The physical conditions, such as structure of substrate, current velocity, etc., of sites S-5, S-6, S-9 and S11 were not preferable for Heptageniidae species. Heptageniidae species mostly prefer high current velocity and rocky substratum (Bauernfeind and Moog, 2002; Buffagni et al., 2009). Therefore, the dominancies of Heptageniidae species were low in these sites in spite of having reference habitat conditions. According to the physico-chemical variables, site S-14 was beta-mesosaprobic (Tab. 4). In this site, there were no physical degradations or any other damaging effects, but the dominancy of Baetidae species was higher than Heptageniidae species. It was assumed that there could have been a degradation in the past and that would have affected the sensitive Heptageniidae species in time.

According to the environmental and statistical results, reference sites were determined and their reference Ephemeroptera communities were given as in table 6. 
Table 4: Reference habitat condition and saprobity status of collecting sites; *RHC, Reference habitat condition.

\begin{tabular}{|l|c|l|l|c|l|}
\hline Sites & RHC $^{*}$ & Saprobity Status & Sites & RHC & Saprobity Status \\
\hline S-1 & - & Alpha-mesosaprobic & S-13 & $\checkmark$ & Oligosaprobic \\
\hline S-2 & - & Beta-mesosaprobic & S-14 & $\checkmark$ & Beta-mesosaprobic \\
\hline S-3 & - & Beta-mesosaprobic & S-15 & - & Beta-mesosaprobic \\
\hline S-4 & $\checkmark$ & Beta-mesosaprobic & S-16 & - & Beta-mesosaprobic \\
\hline S-5 & $\checkmark$ & Beta-mesosaprobic & S-17 & - & Beta-mesosaprobic \\
\hline S-6 & $\checkmark$ & Oligosaprobic & S-18 & - & Oligosaprobic \\
\hline S-7 & $\checkmark$ & Oligosaprobic & S-19 & $\checkmark$ & Oligosaprobic \\
\hline S-8 & $\checkmark$ & Beta-mesosaprobic & S-20 & $\checkmark$ & Oligosaprobic \\
\hline S-9 & $\checkmark$ & Beta-mesosaprobic & S-21 & $\checkmark$ & Beta-mesosaprobic \\
\hline S-10 & $\checkmark$ & Oligosaprobic & S-22 & $\checkmark$ & Beta-mesosaprobic \\
\hline S-11 & $\checkmark$ & Oligosaprobic & S-23 & $\checkmark$ & Beta-mesosaprobic \\
\hline S-12 & $\checkmark$ & Oligosaprobic & S-24 & - & Beta-mesosaprobic \\
\hline
\end{tabular}

Table 5: Species dominancy of Baetidae and Heptageniidae in the collecting sites; *Reference habitats.

\begin{tabular}{|c|c|c|c|c|c|}
\hline Sites & $\begin{array}{l}\text { Dominancy of } \\
\text { Baetidae } \\
\text { species (\%) } \\
\end{array}$ & $\begin{array}{c}\text { Dominancy of } \\
\text { Heptageniidae } \\
\text { species (\%) } \\
\end{array}$ & Sites & \begin{tabular}{|l} 
Dominancy of \\
Baetidae \\
species (\%) \\
\end{tabular} & $\begin{array}{c}\text { Dominancy of } \\
\text { Heptageniidae species } \\
(\%)\end{array}$ \\
\hline S-1 & 73.93 & 3.41 & S-13* & 36.71 & 56.96 \\
\hline S-2 & 30.29 & 6.85 & S-14* & 99.28 & 0.72 \\
\hline S-3 & 65.38 & 6.41 & S-15 & 76.59 & 1.95 \\
\hline S-4* & 43.55 & 55.65 & S-16 & 97.20 & 2.80 \\
\hline$S-5^{*}$ & 62.50 & 37.50 & S-17 & 94.09 & 5.91 \\
\hline S-6 ${ }^{*}$ & 61.37 & 31.52 & S-18 & 88.79 & 11.21 \\
\hline S-7 ${ }^{*}$ & 34.69 & 65.22 & S-19* & 18.31 & 74.13 \\
\hline S-8 ${ }^{*}$ & 20.46 & 77.52 & S-20* & 19.67 & 59.83 \\
\hline$S-9^{*}$ & 70.19 & 22.12 & $\mathrm{~S}-21^{*}$ & 31.40 & 56.07 \\
\hline S-10* & 23.47 & 74.65 & S-22* & 16.43 & 76.95 \\
\hline S-11 & 70.97 & 26.73 & S-23* & 14.66 & 73.29 \\
\hline$S-12^{*}$ & 35.00 & 58.57 & S-24 & 71.00 & 29.00 \\
\hline
\end{tabular}


Table 6: Reference Ephemeroptera communities.

\begin{tabular}{|c|c|}
\hline Ref. sites & Reference Ephemeroptera Communities \\
\hline S4 (Fig. 3) & $\begin{array}{l}\text { Baetis rhodani, Baetis gemellus, Baetis lutheri, Alainites muticus, } \\
\text { Rhithrogena germanica, Epeorus caucasicus, Epeorus alpicola, Epeorus } \\
\text { sylvicola, Epeorus znojkoi, Habroleptoides modesta. }\end{array}$ \\
\hline S5 & $\begin{array}{l}\text { Baetis rhodani, Baetis gemellus, Baetis lutheri, Rhithrogena iridina, Epeorus } \\
\text { caucasicus, Epeorus alpicola, Epeorus znojkoi. }\end{array}$ \\
\hline S6 & $\begin{array}{l}\text { Baetis rhodani, Baetis gemellus, Baetis lutheri, Alainites muticus, } \\
\text { Rhithrogena semicolorata, Rhithrogena zelinkai, Rhithrogena germanica, } \\
\text { Rhithrogena puytoraci, Rhithrogena iridina, Electrogena quadrilineata, } \\
\text { Electrogena affinis, Epeorus caucasicus, Epeorus alpicola, Epeorus } \\
\text { sylvicola, Epeorus znojkoi, Habroleptoides modesta. }\end{array}$ \\
\hline S7 (Fig. 4) & $\begin{array}{l}\text { Baetis rhodani, Baetis gemellus, Baetis lutheri, Alainites muticus, } \\
\text { Rhithrogena semicolorata, Rhithrogena zelinkai, Rhithrogena germanica, } \\
\text { Rhithrogena puytoraci, Rhithrogena iridina, Ecdyonurus starmachi, } \\
\text { Ecdyonurus helveticus, Electrogena quadrilineata, Epeorus caucasicus, } \\
\text { Epeorus alpicola, Epeorus sylvicola, Epeorus znojkoi, Habroleptoides } \\
\text { modesta. }\end{array}$ \\
\hline S8 & $\begin{array}{l}\text { Baetis rhodani, Baetis gemellus, Baetis lutheri, Rhithrogena semicolorata, } \\
\text { Rhithrogena zelinkai, Rhithrogena germanica, Rhithrogena puytoraci, } \\
\text { Ecdyonurus picteti, Ecdyonurus helveticus, Epeorus caucasicus, Epeorus } \\
\text { sylvicola, Epeorus znoikoi, Habroleptoides modesta. }\end{array}$ \\
\hline S9 & $\begin{array}{l}\text { Baetis rhodani, Baetis gemellus, Epeorus alpicola, Epeorus sylvicola, } \\
\text { Habroleptoides modesta. }\end{array}$ \\
\hline S10 (Fig. 5) & $\begin{array}{l}\text { Baetis rhodani, Baetis gemellus, Baetis lutheri, Baetis fuscatus, Baetis milani, } \\
\text { Rhithrogena semicolorata, Rhithrogena zelinkai, Rhithrogena germanica, } \\
\text { Rhithrogena puytoraci, Rhithrogena iridina, Epeorus caucasicus, Epeorus } \\
\text { alpicola, Epeorus sylvicola, Epeorus znojkoi, Habroleptoides modesta. }\end{array}$ \\
\hline S11 & $\begin{array}{l}\text { Baetis rhodani, Baetis gemellus, Baetis lutheri, Baetis fuscatus, Baetis milani, } \\
\text { Rhithrogena zelinkai, Epeorus caucasicus, Epeorus alpicola, Epeorus } \\
\text { sylvicola, Epeorus znojkoi, Habroleptoides modesta. }\end{array}$ \\
\hline S12 & $\begin{array}{l}\text { Baetis gemellus, Baetis fuscatus, Baetis milani, Rhithrogena semicolorata, } \\
\text { Rhithrogena zelinkai, Epeorus caucasicus, Epeorus alpicola, Epeorus } \\
\text { sylvicola, Epeorus znojkoi, Habroleptoides modesta. }\end{array}$ \\
\hline S13 (Fig. 6) & $\begin{array}{l}\text { Baetis rhodani, Baetis gemellus, Baetis lutheri, Alainites muticus, } \\
\text { Rhithrogena semicolorata, Rhithrogena zelinkai, Epeorus caucasicus, } \\
\text { Epeorus alpicola, Epeorus sylvicola, Epeorus znojkoi, Habroleptoides } \\
\text { modesta. }\end{array}$ \\
\hline S14 (Fig. 7) & $\begin{array}{l}\text { Baetis rhodani, Baetis gemellus, Baetis lutheri, Baetis fuscatus, Baetis milani, } \\
\text { Epeorus caucasicus, Epeorus sylvicola, Epeorus zaitzevi. }\end{array}$ \\
\hline S19 (Fig. 8) & $\begin{array}{l}\text { Baetis rhodani, Baetis gemellus, Baetis lutheri, Baetis fuscatus, Alainites } \\
\text { muticus, Baetis milani, Rhithrogena semicolorata, Rhithrogena zelinkai, } \\
\text { Rhithrogena germanica, Rhithrogena puytoraci, Rhithrogena iridina, } \\
\text { Rhithrogena beskidensis, Ecdyonurus picteti, Electrogena affinis, Epeorus } \\
\text { caucasicus, Epeorus sylvicola, Epeorus znojkoi, Serratella ignita, } \\
\text { Habroleptoides modesta. }\end{array}$ \\
\hline
\end{tabular}


Table 6 (continued): Reference Ephemeroptera communities.

\begin{tabular}{|c|l|}
\hline S20 (Fig. 9) & $\begin{array}{l}\text { Baetis rhodani, Baetis gemellus, Baetis lutheri, Baetis fuscatus, Rhithrogena } \\
\text { semicolorata, Rhithrogena zelinkai, Electrogena quadrilineata, Epeorus } \\
\text { caucasicus, Epeorus sylvicola, Epeorus znojkoi, Serratella ignita. }\end{array}$ \\
\hline S21 (Fig. 10) & $\begin{array}{l}\text { Baetis rhodani, Baetis gemellus, Baetis lutheri, Baetis fuscatus, Rhithrogena } \\
\text { semicolorata, Rhithrogena puytoraci, Rhithrogena iridina, Rhithrogena } \\
\text { beskidensis, Electrogena affinis, Epeorus caucasicus, Epeorus sylvicola, } \\
\text { Epeorus znojkoi, Serratella ignita. }\end{array}$ \\
\hline S22 (Fig. 11) & $\begin{array}{l}\text { Baetis rhodani, Baetis gemellus, Baetis lutheri, Alainites muticus, } \\
\text { Rhithrogena semicolorata, Rhithrogena puytoraci, Rhithrogena iridina, } \\
\text { Rhithrogena beskidensis, Epeorus caucasicus, Epeorus sylvicola, Epeorus } \\
\text { znojkoi, Serratella ignita. }\end{array}$ \\
\hline S23 & $\begin{array}{l}\text { Baetis rhodani, Baetis gemellus, Alainites muticus, Rhithrogena } \\
\text { semicolorata, Rhithrogena iridina, Ecdyonurus starmachi, Ecdyonurus } \\
\text { picteti, Ecdyonurus helveticus, Electrogena quadrilineata, Epeorus } \\
\text { caucasicus, Epeorus alpicola, Epeorus znojkoi, Habroleptoides modesta. }\end{array}$ \\
\hline
\end{tabular}

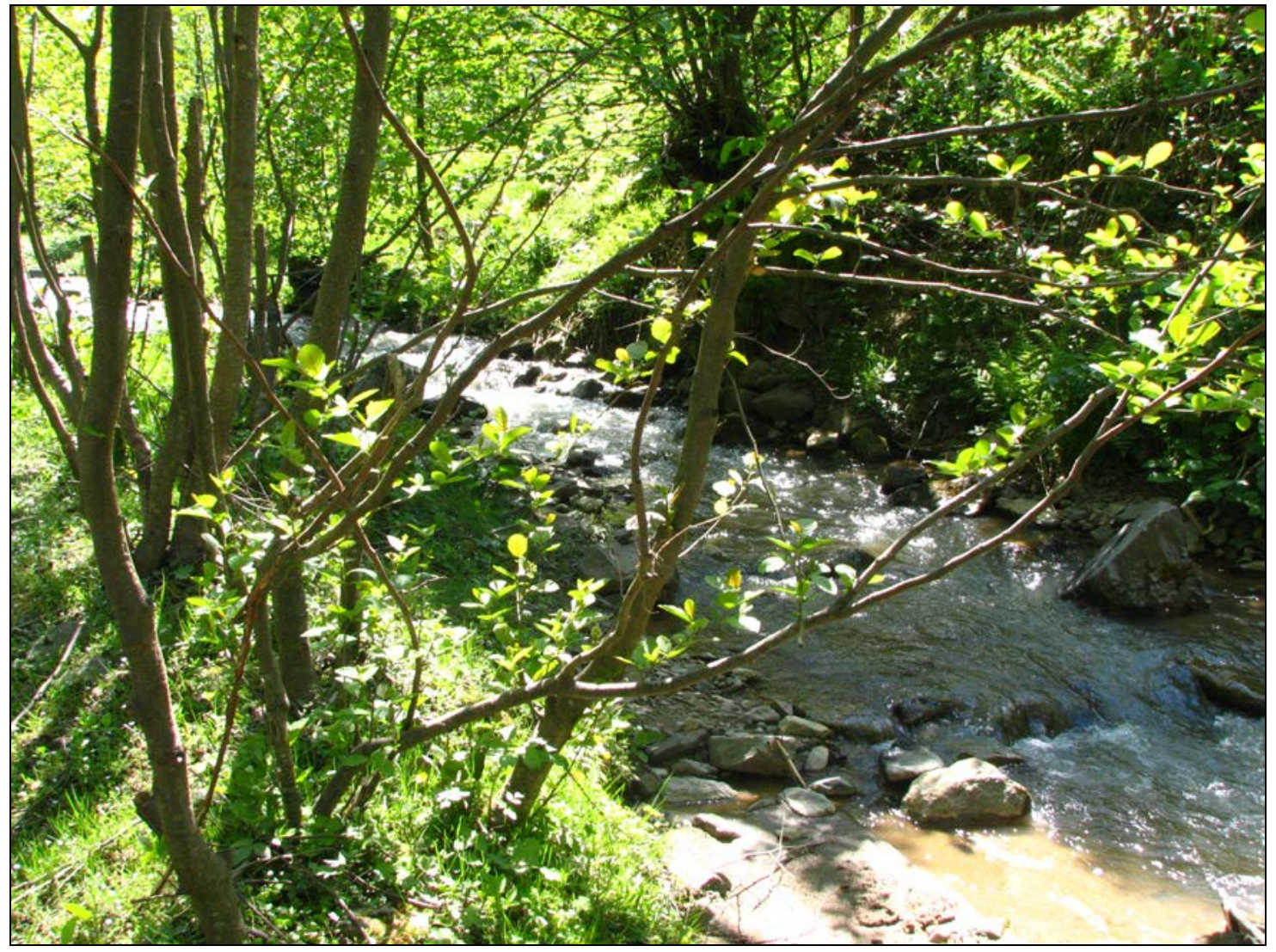

Figure 3: Reference Ephemeroptera community (S4). 


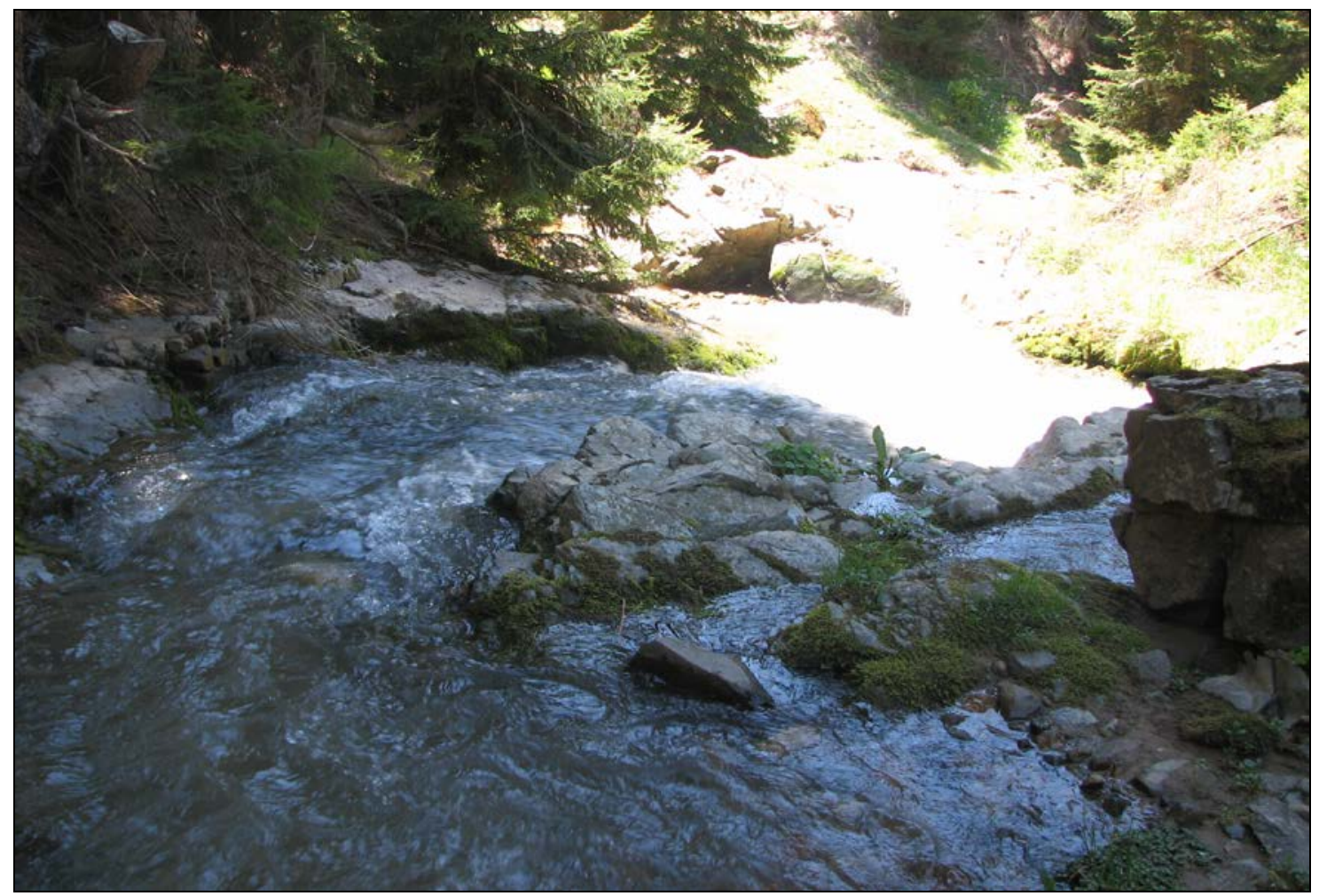

Figure 4: Reference Ephemeroptera community (S7).

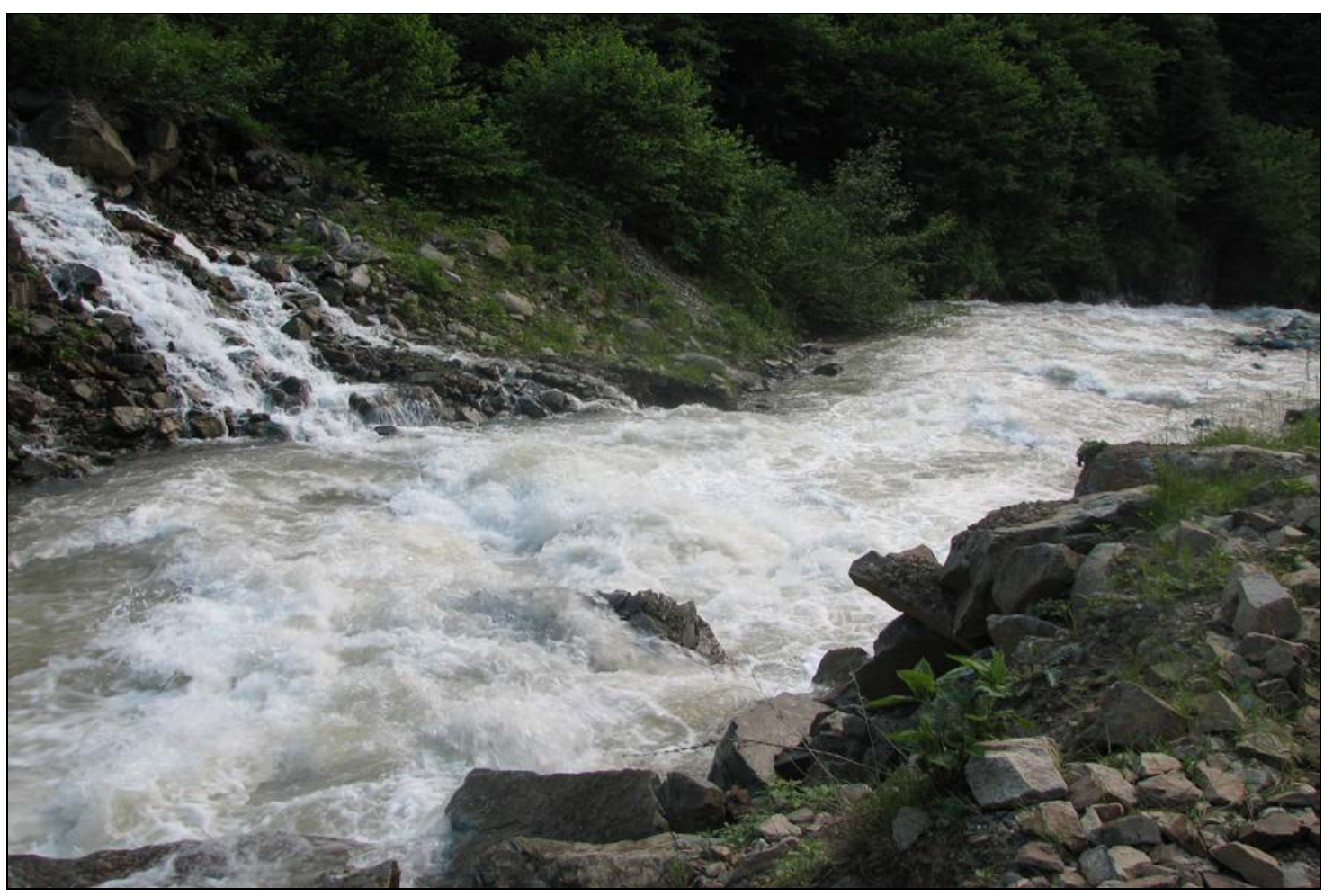

Figure: 5 Reference Ephemeroptera community S10. 


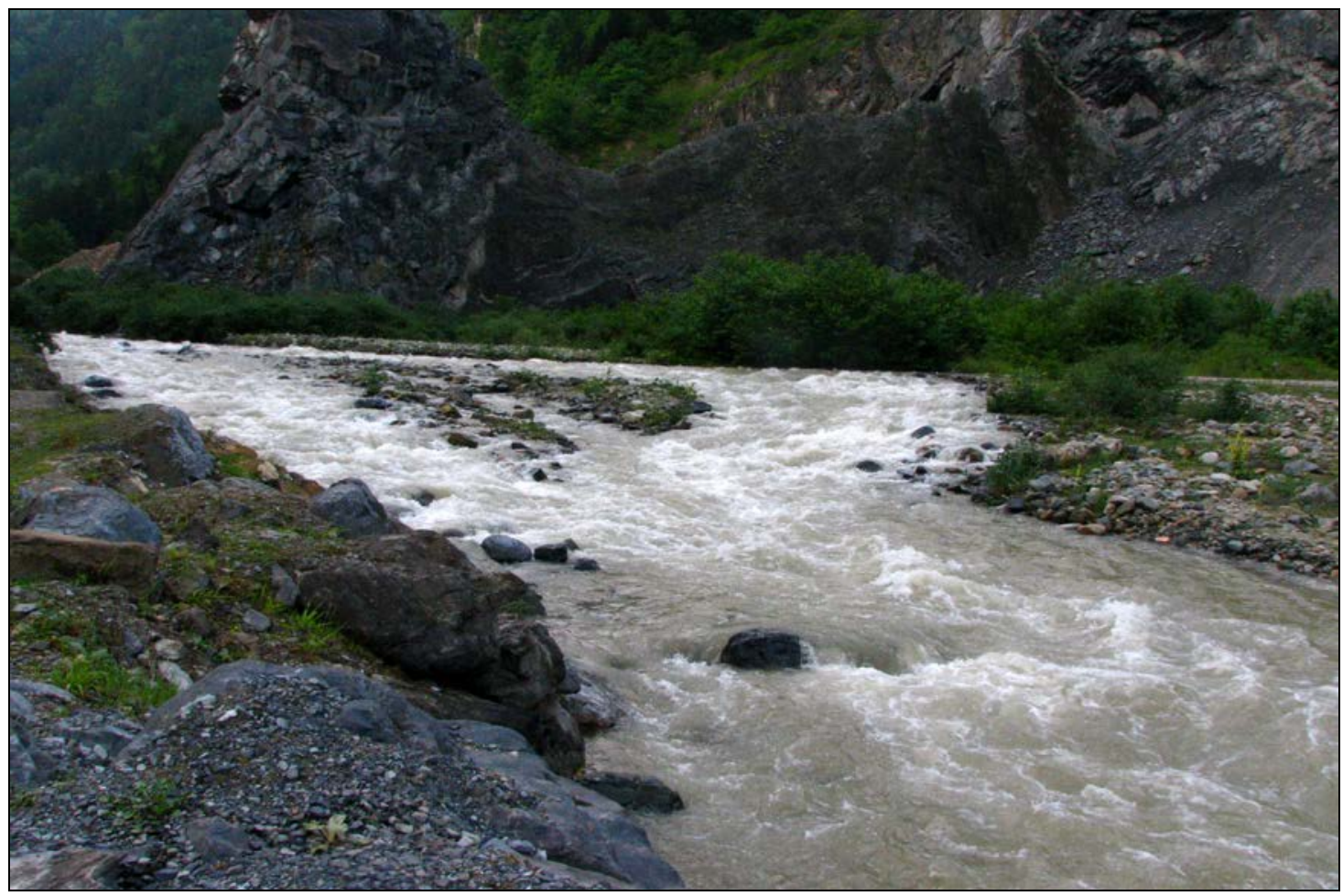

Figure 6: Reference Ephemeroptera community S13.

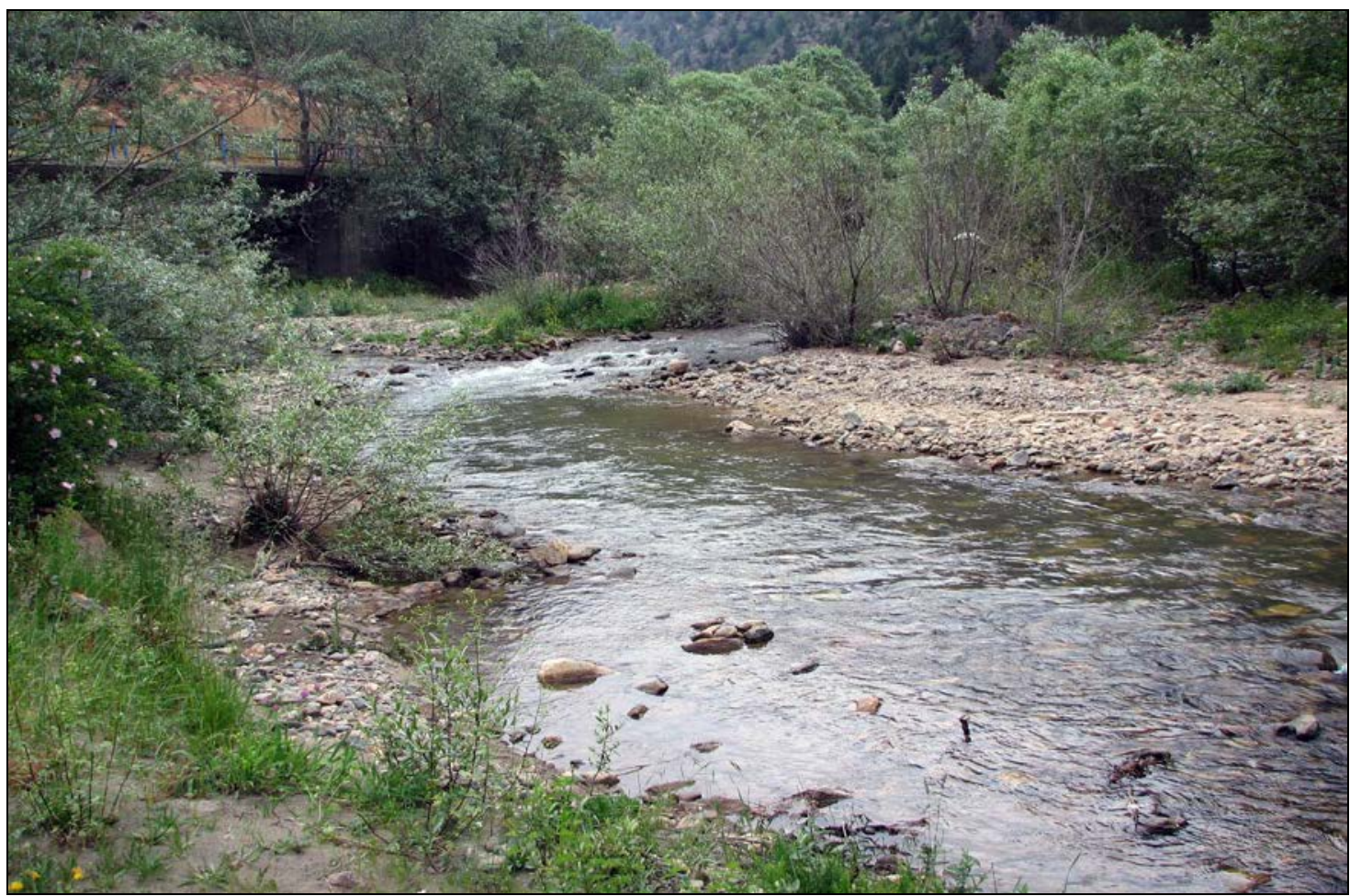

Figure 7: Reference Ephemeroptera community S14. 


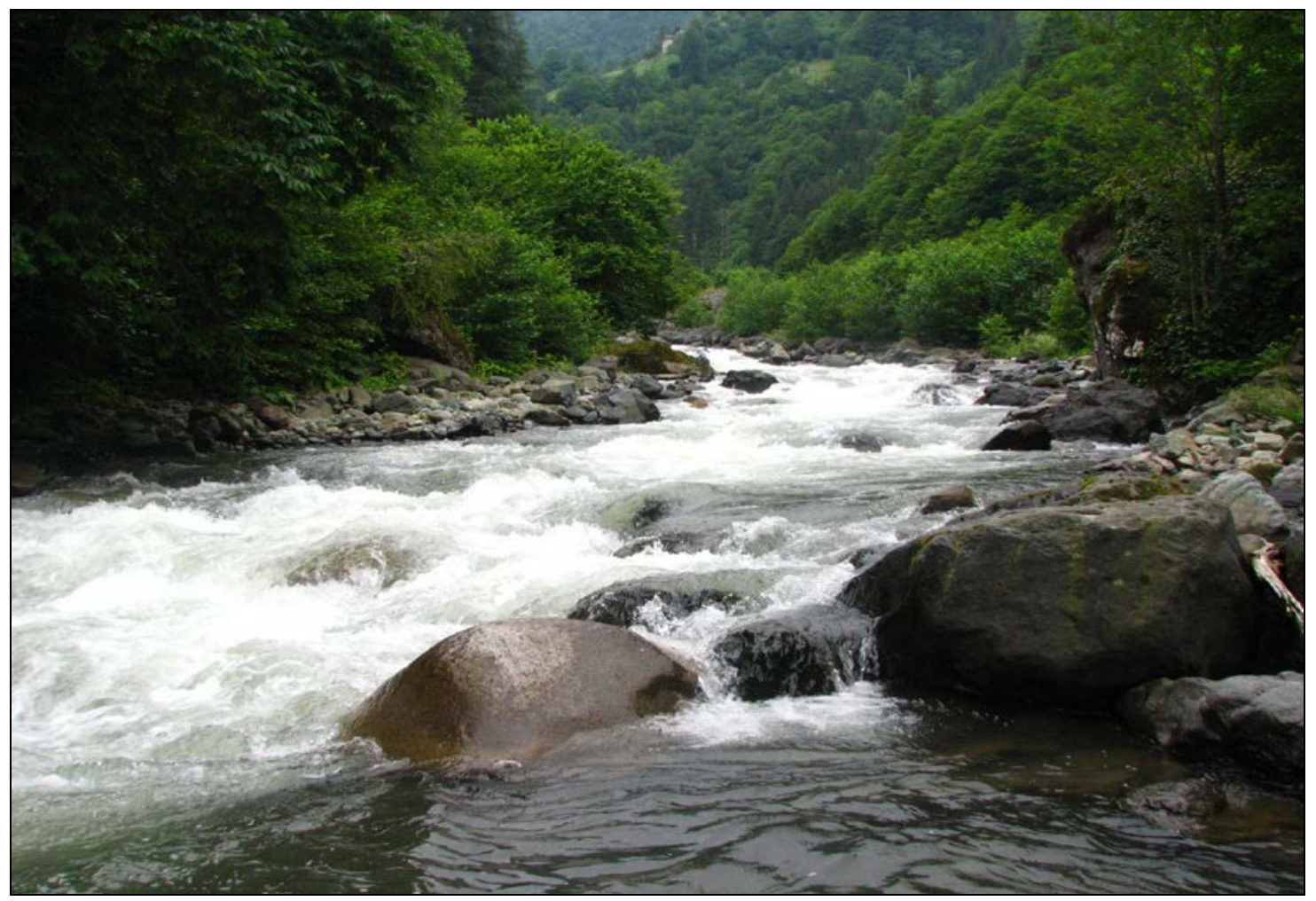

Figure 8: Reference Ephemeroptera community S19.

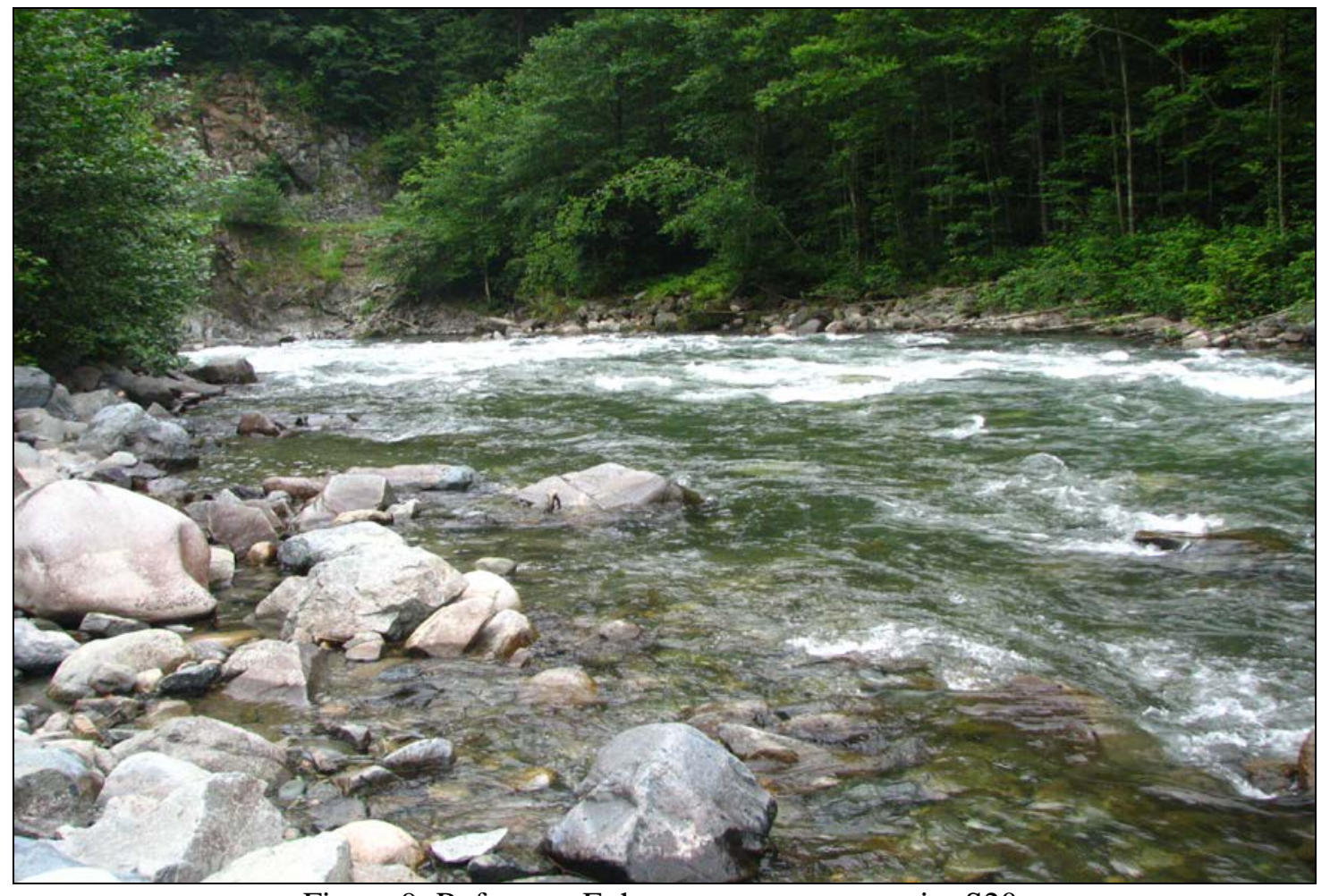

Figure 9: Reference Ephemeroptera community S20. 


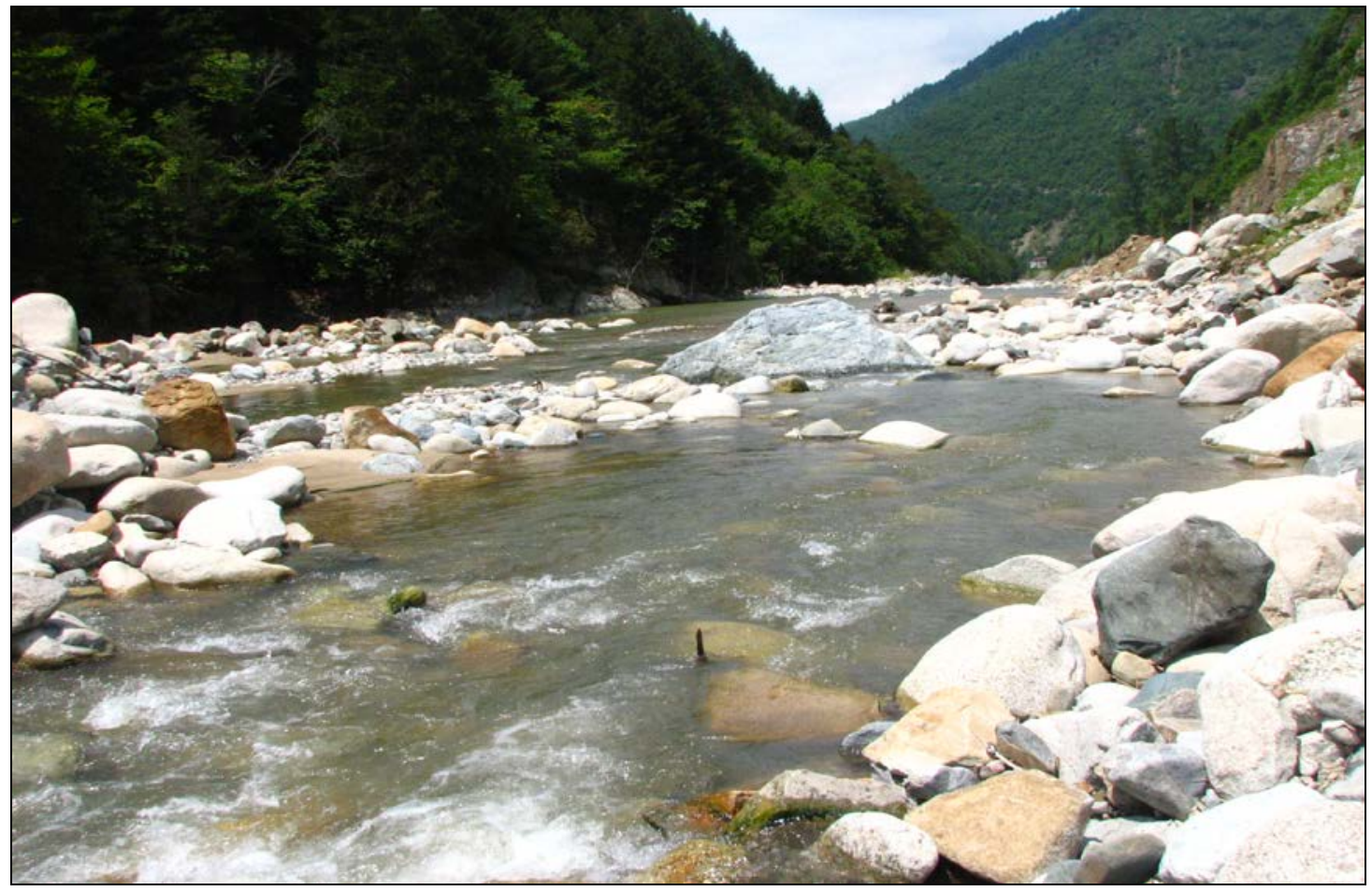

Figure 10: Reference Ephemeroptera community S21.

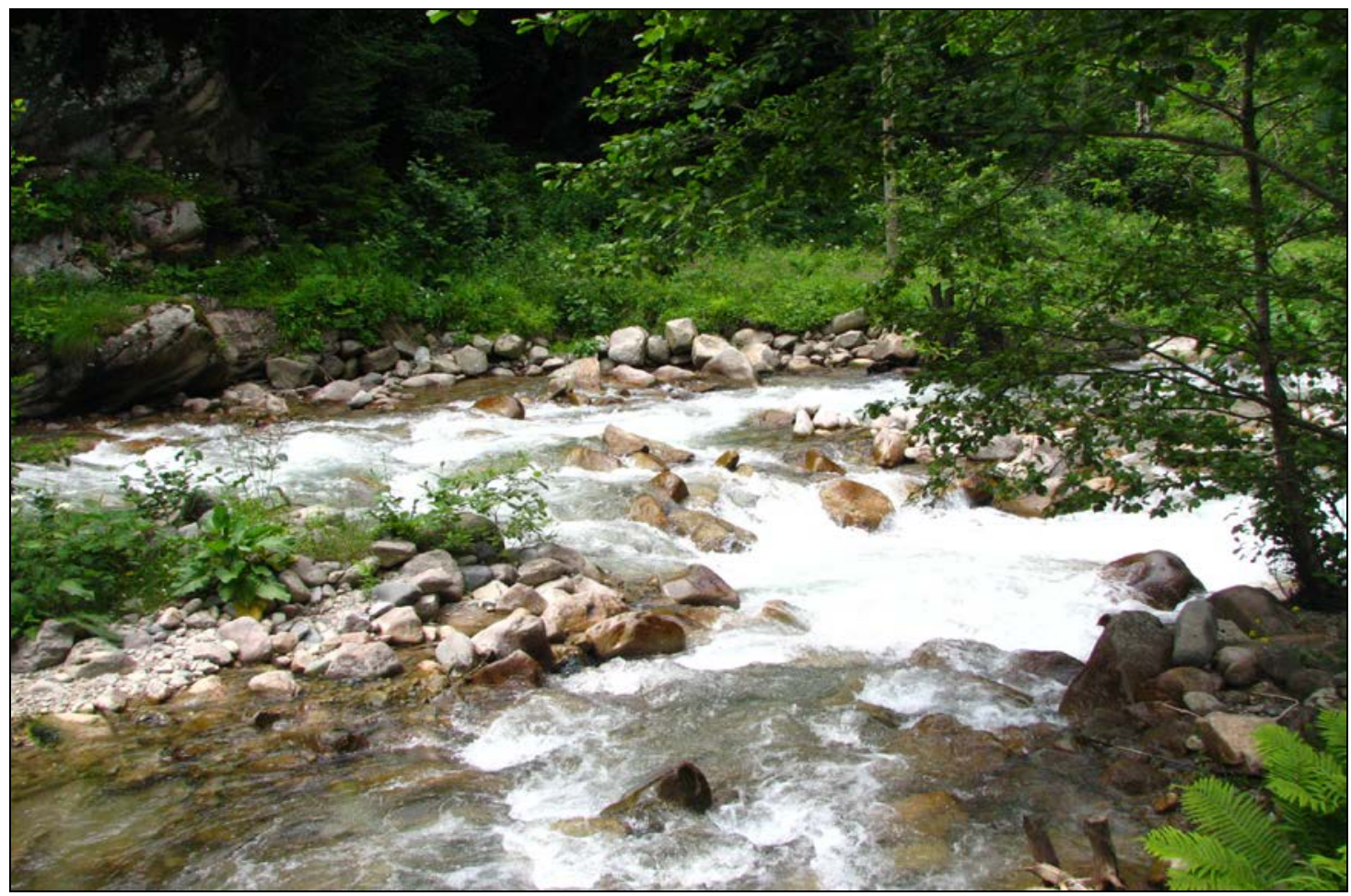

Figure 11: Reference Ephemeroptera community S22. 


\section{CONCLUSIONS}

Determining the reference habitats and reference communities has a great importance in Water Framework Directive. Only through this way is it possible to compare the current situation of running water with the previous one if there was any degradation in the past.

Recently, several hydroelectric power plants were constructed on some running waters in the Eastern Part of Black Sea Region and there are plans to construct several others. These constructions will create a significant effect over the running waters by destroying the natural habitats and causing loss of sensitive Ephemeroptera fauna members in the area. Therefore, it is crucial to clearly determine and show the magnitude of the variation from past to present days by comparing the Ephemeroptera communities. One of the highly emphasized subjects in WFD is the reference habitat condition, because it is necessary to detect how the current status differs from the expected status.

By using the list of reference Ephemeroptera communities, it will be possible to decide which areas have reference habitat condition or how areas are affected from different kinds of degradation.

\section{ACKNOWLEDGEMENTS}

This study was supported by Hacettepe University, Scientific Research Projects Coordination Unit, Project number: 600, Project title: "Systematic and Ecologic Research on the Ephemeroptera Fauna of the Eastern Part of the Black Sea Region to Take Part in the Implementation of Water Framework Directive (WFD)"; Project number: 010 D02 601 004, Project title: "Determination of habitat quality of Aksu Stream (Giresun) by using physicochemical variables and benthic macroinvertebrates based on to European Union Water Framework Directive Criteria”; Project number: 0701601 005, Project title: "Constitution of biotic index for long-term biomonitoring of water quality for Yeşillrmak River by using benthic macroinvertebrates"; Project number: 09 D03 601 002, Project title, "Determining the reference habitats and the Chironomidae (Insecta, Diptera) fauna of these habitats in some running waters in the Eastern Black Sea Region according to the European Union Water Framework Directive Criteria”; and supported by TUBITAK, Project number: TBAG-U/103(104T106), Project title: "Biodiversity of genus Perla important bioindicators in running waters". 


\section{REFERENCES}

1. Bauernfeind E. and Moog O., 2002 - Weichselbaumer P., Ephemeroptera, in Moog O., Fauna Aquatica Austriaca, Lieferung, 2002, Wasserwirtschaftskataster, Bundesministerium für Land- und Forstwirtschaft, Umwelt und Wasserwirtschaft, Wien.

2. Bănăduc A. and Olosutean H., 2013 - The influence of some environmental variables on diversity of Ephemeroptera, Plecoptera and Trichoptera assemblages - Vişeu Basin case study, Transylvanian Review of Systematical and Ecological Research, The Wetlends Diversity, 15.1, 81-90.

3. Buffagni A., Kemp J. L., Erba S., Belfiore C., Hering D. and Moog O., 2001 - A Europe-wide system for assessing the quality of rivers using macroinvertebrates: the AQEM Project and its importance for southern Europe (with special emphasis on Italy), Scientific and legal aspects of biological monitoring in freshwater, Journal of Limnology, 601, 39-48.

4. Buffagni A., Cazzola M., López-Rodríguez M. J., Alba-Tercedor J. and Armanini D. G., 2009 - Distribution and Ecological Preferences of European Freshwater Organisms, Ephemeroptera, 3, Schmidt-Kloiber A. and Hering D. (eds), Pensoft Publishers (SofiaMoscow), 254.

5. Clarke R. T., Wright J. F. and Furse M. T., 2003 - RIVPACS models for predicting the expected macroinvertebrate fauna and assessing the ecological quality of rivers, Ecological Modelling, 3, 219-233.

6. Çoşkun A. A., 2010 - AB Su Çerçeve Direktifi Açısından Türk Hukukunda Nehir Havza Yönetim Planlaması, Süleyman Demirel Üniversitesi Orman Fakültesi Dergisi, A, 1, 43-55. (in Turkish)

7. Council of European Communities, 2000 - Water Framework Directive (WFD) Directive 2000/60/EC of the European Parliament and of the Council of 23 October 2000 establishing a framework for Community action in the field of water policy, Official Journal of the European Communities, L 327, 22.12.2000, 1-72.

8. Hubbard M. D. and Peters W. L., 1978 - Environmental Requirements and Pollution Tolerance of Ephemeroptera, USEPA Report EPA-600/4-78-061, Cincinnati, VI, 461.

9. Kazanc1 N., 2013 - The records of Plecoptera (Insecta) species from Eastern part of Black Sea Region (Turkey), Review of Hydrobiology, 6, 2, 121-127.

10. Kazancı N., Öz B., Türkmen G. and Başören Ertunç Ö., 2011 - Contributions to aquatic fauna of a Biodiversity Hotspot in Eastern Blacksea Region of Turkey with records from runningwater interstitial fauna of some enviromental variables, Review of Hydrobiology, 4, 2, 131-138.

11. Kazancı N., Başören Ö., Türkmen G., Öz B., Ekingen P. and Bolat H. A., 2013 - Assessment of macroinvertebrate community structure and water quality of running waters in Camili (Artvin, Turkey), A part of Caucasus Biodiversity Hotspot, by using Water Framework Directive (WFD) methods, Review of Hydrobiology, 6, 2, 91-102.

12. Kocataş A., 1992 - Ekoloji ve Çevre Biyolojisi, Ege Üniversity Matbaası, İzmir, 564. (in Turkish)

13. Metcalfe J. L., 1989 - Biological water quality assessment of running waters based on macroinvertebrate communities: History and present status in Europe, Environmental Pollution, 60, 101-139.

14. Moog O., Bauernfeind E. and Weichselbaumer P., 1997 - The use of Ephemeroptera as saprobic indicators in Austria, 254-260 in Landolt P, Sartori M. Ephemeroptera and Plecoptera, Biology-Ecology-Systematics, Mauron, Tinguely, Lachat and Fribourg S. A.

15. Odum E. and Barrett G., 2005 - Fundamentals of Ecology, Thomson Brooks/Cole, 598.

16. Rosenberg D. M. and Resh V. H., 1993 - Introduction to Freshwater Biomonitoring and Benthic Macroinvertebrates, Freshwater Biomonitoring and Benthic Macroinvertebrates, 448.

17. Ter Braak C. J. F., 1986 - Canonical correspondence analysis: a new eigenvector technique for multivariate direct gradient analysis, Ecology, 67, 5, 1167-1179. 
18. Türkmen G., 2013 - Systematic and Ecologic Research on the Ephemeroptera Fauna of the Eastern Black Sea Region to Take Part in the Implementation of Water Framework Directive (WFD) Hacettepe University, Science Faculty, Biology Department, PhD Thesis, Ankara, 201. (in Turkish)

19. Türkmen G. and Kazancı N., 2015 - Additional records of Ephemeroptera (Insecta) species from the Eastern Part of Black Sea Region (Turkey), Review of Hydrobiology, 8, 1, 33-50.

20. Van Wijk F. J., De La Hayre M. A. A., Hehenkamp M. J. and Velde I. A., 2003 - Uygulama El kitabı, Su Çerçeve Yönergesinin Türkiye'de uygulanması, MAT01/TR/9/3, Document number: 13/99044324/MJH, Grontmij Consulting Engineers, Houten. (in Turkish) 